\title{
Magneto Optic Current Transformer Technology (MOCT)
}

\author{
Attish Jain \\ Btech, Eee, Srm University Kancheepuram Kattankulathur -603203
}

\begin{abstract}
An accurate electric current transducer is a key component of any power system instrumentation. To measure currents power stations and substations conventionally employ inductive type current transformers .For high voltage applications, porcelain insulators and oil-impregnated materials have to be used to produce insulation between the primary bus and the secondary windings. The insulation structure has to be designed carefully to avoid electric field stresses, which could eventually cause insulation breakdown. The electric current path of the primary bus has to be designed properly to minimize the mechanical forces on the primary conductors for through faults. The reliability of conventional high-voltage current transformers have been questioned because of their violent destructive failures which caused fires and impact damage to adjacent apparatus in the switchyards, electric damage to relays, and power service disruptions. In addition to the concerns, with the computer control techniques and digital protection devices being introduced into power systems, the conventional current transformers have caused further difficulties, as they introduce electromagnetic interference through the ground loop into the digital systems. Magneto-optical current transformer(MOCT)technology provides a solution for many of the above mentioned problems. The MOCT measures the electric current by means of Faraday Effect that is the orientation of polarized light rotates under the influence of the magnetic fields and the rotation angle is proportional to the strength of the magnetic field component in the direction of optical path. MOCT is a passive optical current transducer which uses light to accurately measure current on high voltage systems and determines the rotation angle \& converts it into a signal of few volts proportional to the current.
\end{abstract}

Keywords: electric current transducer, Faraday effect, power system, switchyards, current transformers

\section{MOCT-Principle}

\section{Introduction}

The Magneto-Optical current transformer is based on the Faradays effect. Michael Faraday discovered that the orientation of linearly polarized light was rotated under the influence of the magnetic field when the light propagated in a piece of glass, and the rotation angle was proportional to the intensity of the magnetic field. Generally, this phenomenon can be described as follows:

$\theta=\mathrm{V} \mathrm{dl}$ Eq (1)

' $\theta$ ' is the Faraday rotation angle,

' $\mathrm{V}$ ' is the constant for magneto-optical material

' $\mathrm{B}$ ' is the magnetic flux density along the optical path

' $\mathrm{l}$ ' is the optical path

When the linearly polarized light encircles a current carrying conductor eq(1) can be rewritten as according to Ampere's law as

$\theta=\mathrm{n} \mu \mathrm{VI}$

'I 'is the current to be measured,

' $\mu$ ' is the permeability of the material,

' $n$ ' is the number of turns of the optical path.

The Faraday effect outlined in eq(2) is a better format to apply to an MOCT, because the rotation angle in this case is directly related to the enclosed electric current. It rejects the magnetic field signals due to external currents which are normally quite strong in power system.

A polarizer is used to convert the randomly polarized incident light into linearly polarized light. The orientation of the linearly polarized light rotates an angle $\theta$ after the light has passed through the magnetooptical material because of Faraday Effect. Then another polarization prism is used as an analyzer, which is $45^{\circ}$ oriented with the polarizer, to convert the orientation variation of the polarized light into intensity variation of the light with two outputs, and then these two outputs are send to photo detectors. The purpose of using the 
analyzer is that photo detectors can only detect the intensity of light, rather than the orientation of polarizations. The output optical signals from the analyzer can be described as,

$\mathrm{P} 1=(1+\operatorname{Sin} 2 \theta)$

$\mathrm{P} 2=(1-\operatorname{Sin} 2 \theta)$

$\theta$ is the Faraday rotation angle,

$\mathrm{P} 1$ and $\mathrm{P} 2$ are the optical power delivered by the detectors.

In order to properly apply $\mathrm{Eq}(2)$ in the MOCT design by making the optical path wrap around the current carrying conductor, the optical path has to be folded by reflections. Total internal reflections and metal reflections are good ways to achieve this. However reflections introduce phase shift; hence change the polarization state of the light. The optical prism has to be designed to keep the light going through the MOCT linearly polarized.

\section{System Analysis of MOCT}

The configuration of the test system is shown in block diagram form in the Figure below. The equipment configuration is a current source, adjustable from zero to 4000 amperes, whose output is passed through a precision CT and the MOCT rotator. The output of these two sensors are measured by an instrument package which computes the ratio of the MOCT output with respect to the ct output. This method permits comparison of ratio stability of the MOCT against a known standard. In addition, the ct output is applied to a watt-hour standard while the MOCT output operates the current section of an electronic meter. Application of the current to both the meter under test and the reference standard along with parallel application of a voltage source provides an accurate method of comparing the two systems in a ratio metric manner.

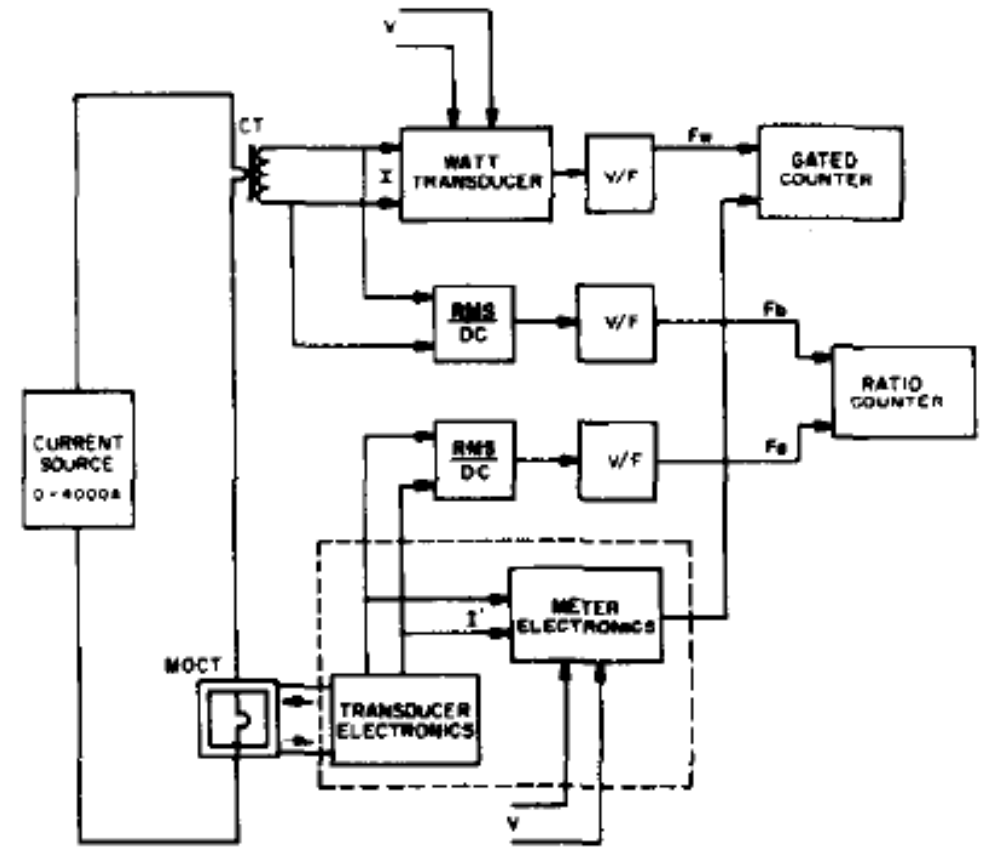

LAB TEST EQUIPAENT

\section{TEST SYSTEM FUNCTIONAL BLOCK DIAGRAM}

\section{Temperature Characteristics}

The first test was to characterize the rotator output with variation in temperature. The rotator and interface electronics were placed in an environmental chamber that allowed temperature profiles to be run on the system while it was operating, and the effect of temperature measured. Two temperature effects were studied. First, the change in the MOCT output that could be related to shifts in the steady state operation due to temperature excursions possible in field conditions. This sets the extremes of the temperature range. Second, it was known that temperature induced stress on the optical system could result in short term changes in the output levels: therefore, a study of the effect of rate of change in temperature was important to characterize the system. 
A typical temperature profile for a test run is obtained. This is considered to be an extreme worst case test condition as it stresses the MOCT system by changing the temperature in a 24 hour period, approximately 10 degrees per hour. Examination of the temperature profiles taken during the field test show this to be a good assumption for the Tennessee area . Following is the variation in ratio performance of the MOCT system under the above test condition. Under this extreme test, the device will exhibit no more than $0.1 \%$ error from $-3 \mathrm{O}^{\circ} \mathrm{C}(-$ $220 \mathrm{~F})$ to $+60^{\circ} \mathrm{C}(+1400 \mathrm{~F})$ when compared to the reference $\mathrm{CT}$ operating at room temperature.

These test were run for each of the precision current transformer ranges with similar results for all ranges. Each test in each range was repeated several time with only slight variations in results from test to test.

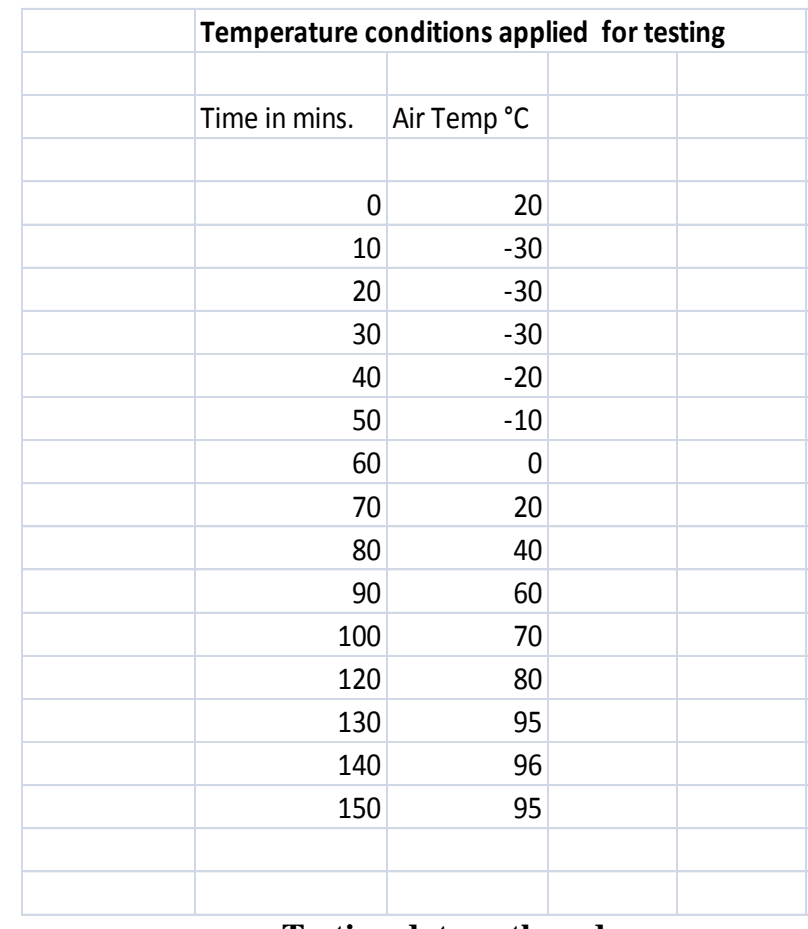

Testing data gathered

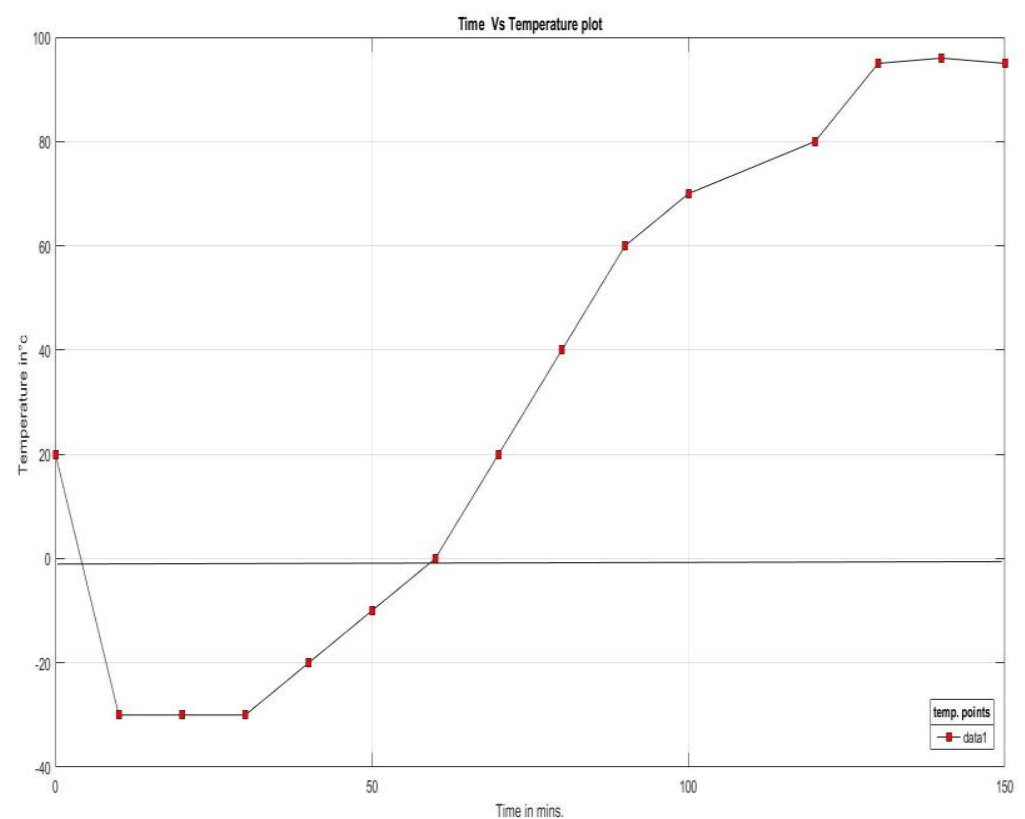

Simulation done on Matlab for temperature vs. time data 


\begin{tabular}{|c|c|c|}
\hline \multicolumn{3}{|c|}{ MOCT and conventional ct comparison Stability Ratio } \\
\hline Temperature in ${ }^{\circ} \mathrm{C}$ & MOCT stability ratio & Conventional ct ratio \\
\hline-20 & 1 & 1 \\
\hline-10 & 1 & 1 \\
\hline 0 & 1 & 1 \\
\hline 10 & 1 & 1 \\
\hline 20 & 0.999 & 0.999 \\
\hline 30 & 0.999 & 0.998 \\
\hline 40 & 0.997 & 0.995 \\
\hline 50 & 0.998 & 0.995 \\
\hline 60 & 0.996 & 0.996 \\
\hline 70 & 0.997 & 0.994 \\
\hline
\end{tabular}

Temperature vs stability ratio factor for a MOCT system and conventional ct system

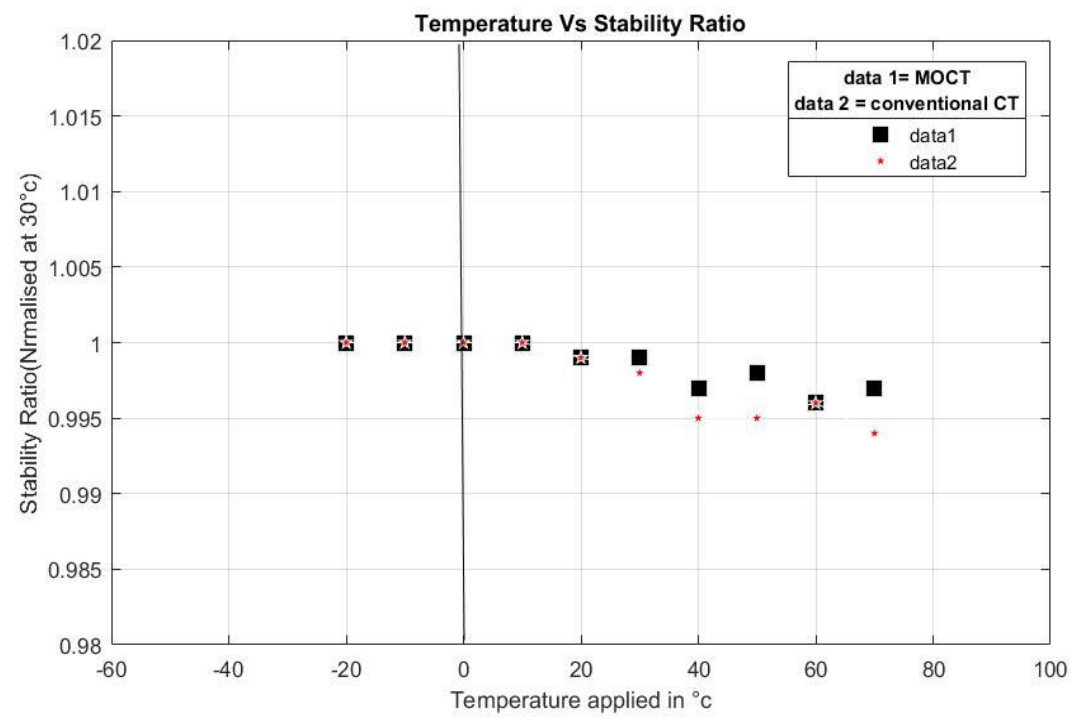

Matlab simulation for Temperature vs stability ratio factor for a MOCT system and conventional ct system

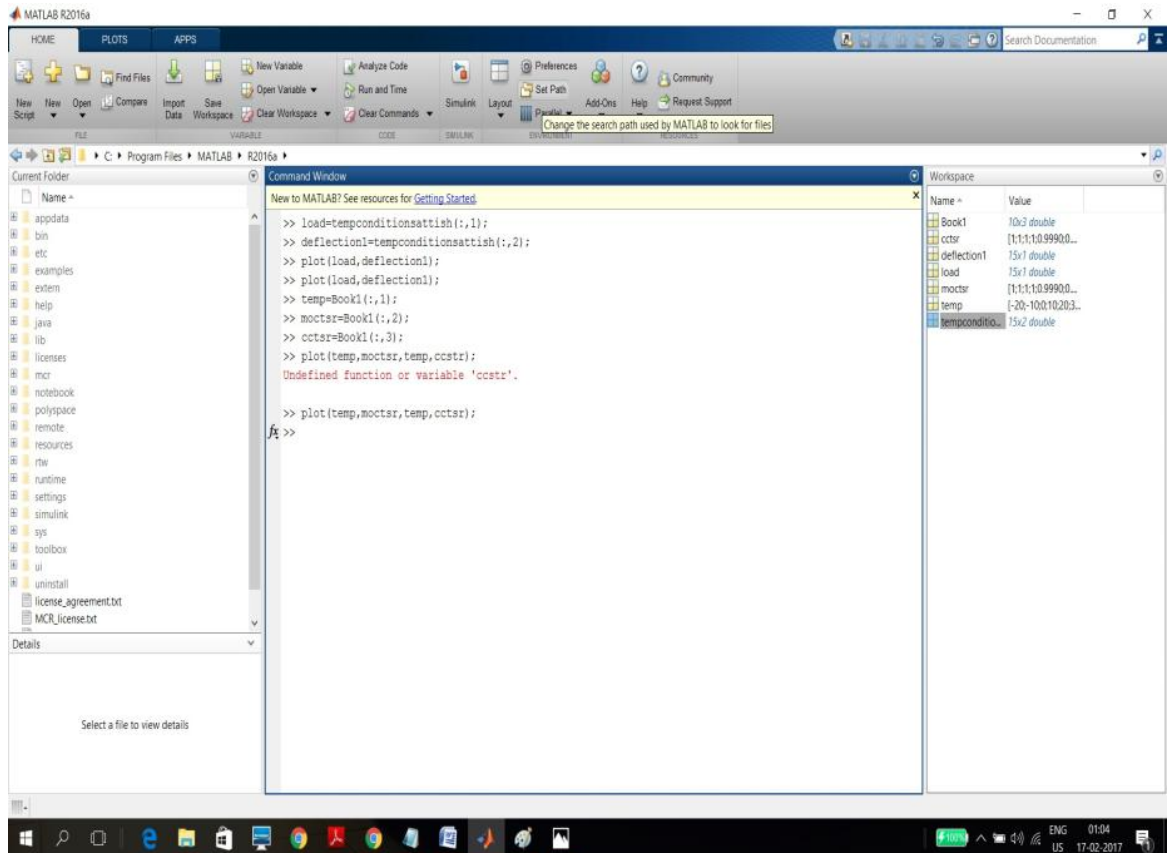

Matlab code for simulation of temperature and stability coefficient ratio 


\section{Advantages of a MOCT system over a conventional ct system:}

1. Resistance to Acoustic and Electromagnetic interferences is excellent which plays an important role in protecting the equipment

2. In terms of size and weight, they have small size and low weight ratio. The probable installation and relocation does not require heavy machinery which makes it economical

3. They have high electrical insulation resistance.

4. Lack of magnetic saturation. Due to Lack of core, there is no saturation which solves many protection and Measurement problems

5. Lack of Ferro resonance phenomena and hysteresis.

6. The measurement error is less, than $0.3 \%$.

7. The power supply is not used in the HV section.

8. Self-supervision is possible.

9. They can withstand high mechanical load.

10. Complete isolation in high voltage section and control room.

11. They are compatible with computer systems and can provide output in digital format.

12. They provide protection against short circuits and output breaks

13. PCB that are severe environmental pollutants are not used.

14. The measurement and analysis of current with higher harmonics is possible.

\section{Conclusion}

The results have confirmed that the MOCTs are suitable for power system protection and can replace the current magnetic CTs used in the industries as the variation of stability coefficient/factor analysed is always close to 1 for various test temperature scale applied on the MOCT apparatus. As can be seen the MOCT system compares favourably with the conventional system. In terms of comparison of performance against objectives for the project, the MOCT demonstrate the following applications/advantages over conventional current transformer:

* Optical sensors can be designed to perform reliably in operating system environments

* Stable and accurate performance in the substation environment is obtainable with proper design

* Effects of temperature, humidity, vibration, and electromagnetic interference are negligible or nonexistent when the system is compared to a high accuracy conventional system

In future the MOCT technology can be used for advanced tariff measurement and protective relays . Values of RMS of Output current in conventional CTs and MOCT are measured by less than $0.1 \%$ difference from each other which relates to the high quality of the MOCT output signal MOCT can determine the future output of the signal and hence can replicate the given signal if a given portion of a signal is lost thereby giving a continuous and uninterrupted signal at the output end. Rapid advances in the quality of performance and costs of the optical fibre and electronic equipment encourage development of measuring trances based on new technology. There is a high scope of application in the field of transmission and distribution for MOCT technology which would result in safer and more cost efficient systems.

\section{References}

[1]. IEEE Transactions on Power Delivery, Vol. 5, No. 2, April 1990, Paul Johnston

[2]. Westinghouse Electric Corporation Raleigh, North Carolina

[3]. S. Saito, J. Hamasaki, Y. Fujii, K. Yokoyama, and Y. Ohno, "Development of the laser current transformer for extra high- voltage power transmission lines', IEEE J. Quant. Elec., vol QE-3, pp. 569-597, Nov. 1967.

[4]. A.J. Rogers, "Method for the simultaneous measurement of current and voltage on high-voltage lines using optical techniques," Proc. lEEE, Vol. 123, pp. 957-960, Oct. 1976.

[5]. S.C. Rashleigh and R. Ulrich, "Magneto-optic current sensing with bi fringing fibres', Appl. Phys. Lett., vol. 34, pp. 766-770, June 1979.

[6]. D.C. Erickson, "The use of fibre optics for communications, measurement, and control within high voltage substations,' IEEE Trans. on Pwr. Appr. and Syst., vol. PAS-99, pp. 1057-1065, May/June 1980.

[7]. R.H. Stolen and E.H. Turner, "Faraday rotation in highly birefringent fibers", Appl. Opt., vol. 19, pp. 842-845. 15 March 1960.

[8]. G.w. Day, D.N. Payne, A.J. Barlow, and J.J. Ramskov Hanson, "Faraday rotation in coiled, monomode optical fibers: Isolators, filters, and magnetic sensors', Opt. Lett., vol. 7, pp. 238-240, May 1982.

[9]. M. Kanoi, G. Takahashi, T. Sato, M. Higaki, E. Mori, and K. Okumura, 'Optical voltage and current measuring system for electric power systems', IEEE Trans. on Pwr. Del., vol. PVVRD-1, pp. 91-97, Jan. 1986.

[10]. P.R. Forman and F.C. Jahoda, "Linear birefringence effects on fiber-optic current sensors", App. Opt., vol. 27, pp. 